\title{
Enhanced Low-Latency Detection of Motor Intention From EEG for Closed-Loop Brain-Computer Interface Applications
}

\author{
Ren Xu, Student Member, IEEE, Ning Jiang, Member, IEEE, Chuang Lin, Natalie Mrachacz-Kersting, \\ Kim Dremstrup, Member, IEEE, and Dario Farina*, Senior Member, IEEE
}

\begin{abstract}
In recent years, the detection of voluntary motor intentions from electroencephalogram (EEG) has been used for triggering external devices in closed-loop brain-computer interface (BCI) research. Movement-related cortical potentials (MRCP), a type of slow cortical potentials, have been recently used for detection. In order to enhance the efficacy of closed-loop BCI systems based on MRCPs, a manifold method called Locality Preserving Projection, followed by a linear discriminant analysis (LDA) classifier (LPP-LDA) is proposed in this paper to detect MRCPs from scalp EEG in real time. In an online experiment on nine healthy subjects, LPP-LDA statistically outperformed the classic matched filter approach with greater true positive rate $(79 \pm 11 \%$ versus $68 \pm 10 \% ; p=0.007)$ and less false positives $(1.4 \pm 0.8 / \mathrm{min}$ versus $2.3 \pm 1.1 / \mathrm{min} ; p=0.016)$. Moreover, the proposed system performed detections with significantly shorter latency $(315 \pm$ $165 \mathrm{~ms}$ versus $460 \pm 123 \mathrm{~ms} ; p=0.013$ ), which is a fundamental characteristics to induce neuroplastic changes in closed-loop BCIs, following the Hebbian principle. In conclusion, the proposed system works as a generic brain switch, with high accuracy, low latency, and easy online implementation. It can thus be used as a fundamental element of BCI systems for neuromodulation and motor function rehabilitation.
\end{abstract}

Index Terms-Brain-computer interface, electroencephalogram (EEG), Locality Preserving Projection, motor intention, movement-related cortical potentials (MRCP).

Manuscript received September 3, 2013; revised October 29, 2013 and November 25, 2013; accepted November 29, 2013. Date of publication December 5, 2013; date of current version January 16, 2014. This work was supported financially by the EU project BETTER (Contract Nr. 247935), and by China Scholarship Council (Contract Nr. 201204910155). Asterisk indicates corresponding author.

R. Xu and N. Jiang are with the Department of Neurorehabilitation Engineering, Bernstein Focus Neurotechnology Göttingen, Bernstein Center for Computational Neuroscience, University Medical Center Göttingen, Georg-August University, 37075 Göttingen, Germany (e-mail: ren.xu@bccn.uni-goettingen. de; ning.jiang@bccn.uni-goettingen.de).

C. Lin is with the Department of Neurorehabilitation Engineering, Bernstein Focus Neurotechnology Göttingen, Bernstein Center for Computational Neuroscience, University Medical Center Göttingen, Georg-August University, 37075 Göttingen, Germany. He is also with with School of Software, Dalian University of Technology, Dalian 116023, China (e-mail: lin.chuang@bccn. uni-goettingen.de).

N. Mrachacz-Kersting and K. Dremstrup are with the Center for SensoryMotor Interaction, Department of Health Science and Technology, Aalborg University, DK-9220 Aalborg, Denmark (e-mail: nm@hst.aau.dk; kdn@hst. aau.dk).

${ }^{*} \mathrm{D}$. Farina is with the Department of Neurorehabilitation Engineering, Bernstein Focus Neurotechnology Göttingen, Bernstein Center for Computational Neuroscience, University Medical Center Göttingen, Georg-August University, 37075 Göttingen, Germany (e-mail: dario.farina@bccn.uni-goettingen.de).

Color versions of one or more of the figures in this paper are available online at http://ieeexplore.ieee.org.

Digital Object Identifier 10.1109/TBME.2013.2294203

\section{INTRODUCTION}

A $\mathrm{N}$ electroencephalogram (EEG) signal based braincomputer interface $(\mathrm{BCI})$ provides an alternative communication channel for healthy or disabled users from their brain to the external environment, bypassing the physiological neural pathways (i.e., peripheral nerves and muscles). In the past decades, BCI has been successfully developed as an alternative communication tool for locked-in patients [1]. More recently, BCI has emerged as a novel tool for close-loop control for patients who suffered, e.g., a stroke or spinal-cord injury [2]. These closed-loop applications are either for mobility assistance, such as controlling a wheelchair [3], or for neurorehabilitation applications, such as controlling an electrical stimulator (ES) [4], [5]. For these purposes, the motor intentions of the user, either imagery, execution or attempt, need to be detected from brain signals with very short delay (several hundred $\mathrm{ms}$ ), so that the resulting control can be perceived as a closed loop. For rehabilitation applications in particular, the afferents resulting from the peripheral stimulation triggered by the $\mathrm{BCI}$ detections have to reach the task specific cortical area within a very narrow time window so that the underlying Hebbian principle can be satisfied, and the rehabilitation can be effective [6].

Two noninvasive motor-related cortical signal modalities have been investigated for closed-loop BCI in neurorehabilitation: sensory motor rhythms (SMR) [7] and movementrelated cortical potentials (MRCP) [8]. SMR-based systems could achieve reasonable detection accuracy, but the latency (i.e., the delay between the motor intention and the corresponding detection) was in the order of seconds [9], [10], which is hardly enough for the Hebbian principle to be effective [6]. This might be one of the key reasons that SMR-based studies reported long intervention protocol (weeks even months) were necessary before any physiological and functional changes could be observed in stroke patients [11], [12]. On the other hand, in a series of recent studies on healthy subjects and stroke patients, it has been established that motor intentions can be detected through MRCP with a latency short enough to elicit fast evolving cortical excitability by triggering peripheral ES [13]-[15]. For clarifying the terminology, it is worth mentioning that the MRCP is referred to contingent negative variation $(\mathrm{CNV})$ when generated in a synchronized (cue-based) paradigm [16], and Bereitschafts potential (BP) or readiness potential when generated in an asynchronized (self-paced) paradigm [17].

In previous studies on closed-loop BCIs based on MRCPs, both $\mathrm{CNV}$ and $\mathrm{BP}$ were investigated [6], [18]-[22]. In a recent 
study by Niazi et al. [13], the MRCP was detected by a simple matched filter (MF), with supervised extraction of the MRCP template. This approach was a satisfactory balance between detection accuracy and latency [13], [14], [23]. Furthermore, as demonstrated by Niazi et al. [14], the induced plasticity is positively associated with the detection accuracy. Therefore, the accuracy and delay of the user motor intention detection algorithm have a direct impact on the efficacy of this approach, or any other approaches, in inducing neural plasticity for rehabilitation training.

The MF is optimal for maximizing the signal-to-noise ratio when the noise is additive Gaussian. This condition is not necessarily valid in the current application. Thus, the MF is very likely to be suboptimal for this application. In the current study, a manifold learning algorithm, called Locality Preserving Projections (LPP) [24], followed by a linear discriminant analysis (LDA) classifier is proposed for the detection problem in MRCP-based BCIs. Furthermore, an online BCI experiment was designed to compare the performance in detection accuracy and latency of the proposed algorithm with that of the MF approach. The results showed that the proposed system works as a generic brain switch, with high accuracy, low latency, and easy online implementation. Given its high performance as a brain switch, it is a critical step in the development of a highly effective, closedloop BCI system for applications such as neuromodulation and motor function rehabilitation.

\section{METHODS}

\section{A. Experimental Setup}

Nine channels of EEG were collected using an active EEG electrode system (ActiveCAP, Brainproducts) and the g.USBamp amplifier (gTec, $\mathrm{GmbH})$ at the sampling rate of $1200 \mathrm{~Hz}$. Nine signal electrodes were placed on Cz, Fz, FC1, $\mathrm{FC} 2, \mathrm{C} 3, \mathrm{C} 4, \mathrm{CP} 1, \mathrm{CP} 2$, and $\mathrm{Pz}$, while the ground electrode and reference electrode were placed on $\mathrm{AFz}$ and the left earlobe, respectively, according to the standard international 10-20 system. The necessary electrode preparation was done so that the impedances of all electrodes were below the recommended value by the manufacturer of actiCAP system before data acquisition.

One channel of surface electromyography (EMG) was also recorded with the g.USBamp amplifier. EMG was acquired in monopolar montage from the tibialis anterior (TA) muscle with disposable electrodes (Neuroline 720, Ambu). The electrode was placed on the mid-belly of the right TA muscle, while the reference and ground electrodes were placed on the bony surface of the right knee and right ankle, respectively.

The nine EEG channels were connected to the first 9 inputs of the g.USBamp amplifier, while the EMG to the last input. The ground and reference setting was done through software configuration, so that the EEG channels had common ground and reference, while the EMG channel had separate ones.

The force of dorsiflexion was recorded using a custommade pedal with force transducer (Aalborg University, Aalborg, Denmark). The force signal was recorded by National Instrument data acquisition card (NI USB-6251), and was presented to the subject, using a graphic user interface (GUI) in MATLAB.

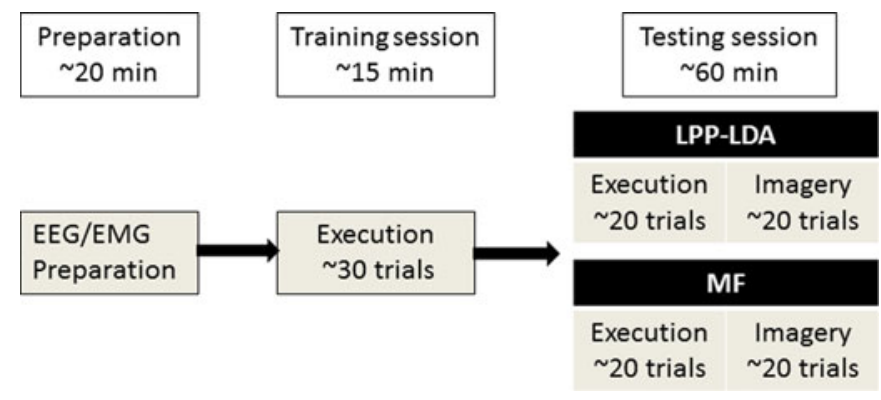

Fig. 1. Experiment Flow. After preparation, the experiment started with a training session and a testing session in which self-paced ME and MI with LPP-LDA and MF were performed. In the last part the experiment, the order of the LPP-LDA runs and MF runs was randomized.

\section{B. Experimental Procedures}

An online experiment was executed to test the validity of the proposed method since online performance is more relevant and closer to real-life applications than offline analysis. The online experiment was executed on nine healthy subjects (age: $29.7 \pm$ $4.3 \mathrm{yrs}$ ) and consisted of three sessions: preparation, training, and testing (see Fig. 1). The experiments were approved by the local ethical committee and the subjects signed an informed consent form before participation.

1) Preparation: In the preparation session, EEG and EMG electrodes were mounted according to the experimental setup described previously. The subjects were asked to perform ballistic dorsiflexions of their right ankle. The torque produced at the ankle joint was acquired and displayed in a subject-view GUI. In the beginning of each experiment, the subjects were instructed to perform a maximum voluntary contraction (MVC) for $10 \mathrm{~s}$. Then, the target contraction level in the training session was set between 40-60\% of MVC. A vertical bar was displayed on the subject-view GUI for feedback purpose in the training session. The height of the bar was proportional to the torque value. The bar was green when the torque was within the target range and blue (red) when it was below (above) the target.

2) Training: Once the target torque was set, the training session started. The subjects were asked to perform self-paced motor execution, i.e., real movements of dorsiflexions, with resting intervals of 10-20 s. Upon the detection of each movement from the EMG (see Section II-D.1), an interval starting $2.5 \mathrm{~s}$ before and terminating $1.5 \mathrm{~s}$ after the movement onset of the preprocessed virtual Cz channel (see detail in Section II-D.2) was displayed to the experimenter. Corrupted trials were flagged by the experimenter, and excluded from further processing (see details in Section II-D).

There are two main reasons why real movements rather than cue-based imagery were used for the training set. First, the signal-to-noise ratio is higher for MRCP in real than in imagined movements [25]. Second, the MRCPs obtained from real movement can be aligned exactly by detecting the movement onset from the EMG, whereas there is an unpredictable alignment error for motor imagery. The use of real movements for training would be possible for patients who have residual muscle activity. Recently, high detection performance of voluntary movement 
onset was reported using EMG contaminated by spasm from stroke patients [26]. In our previous studies using MF on stroke patients, surface EMG from TA muscles was successfully used for the onset detection purpose [13], [27]. Cue-based imagery would be needed for patients without any residual activity, though.

In the training session, the subjects were instructed to perform 30 successful executions. This number was chosen based on previous studies [13], [14], as such reasonable length of repeated dorsiflexions would not make the TA muscle fatigue and thus the subjects could keep full concentration. It would be very difficult for stroke patient to stay alert and concentrate when the intervention protocol is too long. Because some executions were flagged as corrupted, the actual number of executions was usually slightly greater than 30 . There were short rests upon the subjects' requests. The data acquired in the training session were stored, and used to train the LPP-LDA classifier and the MF detector (see Section II-D).

3) Testing: Once the classifiers were trained, the testing session started. Two runs (continuous recording) were performed in this session: LPP run and MF run, for the two detectors. In each run, the subjects performed first motor executions and then imagery. The order of the two runs was randomized.

The execution tasks represents a test close to the real-life applications on patient with residual muscle activity while the imagery tasks represented the situation of patients who cannot execute any movement. Therefore, both of these tasks were carried out in the testing session and used to investigate the performance of the proposed method in detecting motor intentions from EEG.

Both LPP-LDA and MF were applied on execution and imagery (see Fig. 1). During execution, the subject was asked to perform self-paced dorsiflexions, as was done in the training session, for 15-20 times. During imagery, the subjects were asked to perform self-paced dorsiflexion imagery and to lightly say "YES" $1 \mathrm{~s}$ after each imagery task in order to have a reference for the motor imagery events. In a preliminary investigation, it was determined that light simple speech did not influence the proper functioning of the two detectors. A red bar appeared in the subject-view GUI every time a detection occurred (either true or false detection). Both executions and imagery in the testing session were referred to as active phases. There was a passive phase before each active phase, in which the subjects were requested to totally relax for $1 \mathrm{~min}$ without any movement or motor imagery of the TA muscle. Normal eye movements, mouth movements, or slight trunk movement was allowed. This was to test the ability of the algorithms in rejecting potential artifacts produced by these factors under a realistic condition. In this phase, the number of passive false positive ( $\mathrm{pFP}$ ) was counted for $1 \mathrm{~min}$.

During the testing session, the true positive rate (TPR), active false positive (aFP) and pFP were calculated online. The TPR was the ratio of true detections and total attempts, which were identified from the EMG channel for execution tasks and from subject's verbal indication for imagination tasks. The aFP was the number of false detection per minute in the active phases and $\mathrm{pFP}$ was the number of false detections per minute in the passive phases. Additionally, the detection latency (DL) was calculated offline. DL was the latency between the time of the detection and the EMG onset of corresponding movement for the executed tasks. For imagery, however, there was no EMG reference and thus the reference instant for latency calculation was the negative peak of the MRCP, which corresponds to the beginning of motor execution for executed tasks [28].

\section{Locality Preserving Projection}

The algorithm of LDA is well known in the literature, and have been successfully applied in EEG signal classification [29], [30]. Therefore, for the sake of conciseness, the detail of LDA is not repeated here. On the other hand, although LPP has been successfully used in applications such as image processing [31], it has not been applied to EEG signal processing in the context of MRCP detection. Thus, the method is presented in the following.

LPP, as a manifold learning dimensional reduction algorithm, has the property that it preserves the local intrinsic structure of the data in the original high dimension. Given a graph $G$ with $N$ vertices (each vertex represents a training sample), let $\mathbf{W}$ be a $N \times N$ symmetric matrix with each $W_{i j}$ representing the distance between vertices $i$ and $j$, i.e., $W_{i j}=\exp \left(-\left\|x_{i}-x_{j}\right\|^{2} / t\right.$. Here, $t$ is a constant. Then, $G$ and $\mathbf{W}$ can be defined to characterize certain statistical or geometric property of the training samples.

Consider the problem of mapping samples on the graph $G$ to a line so that the connected points are as close as possible. Let $\boldsymbol{y}=\left[y_{1}, y_{2}, \ldots, y_{N}\right]^{T}$ be such a map. The optimal $\boldsymbol{y}$ is given by

$$
\min \sum_{i \cdot j}\left(y_{i}-y_{j}\right)^{2} W_{i j}
$$

Minimizing (1) corresponds to ensuring that if the vertices $i$ and $j$ are "close," then the projections $y_{i}$ and $y_{j}$ are close as well [31], which explains the term "locality preserving." With algebraic formulations, we obtain

$$
\sum_{i . j}\left(y_{i}-y_{j}\right)^{2} W_{i j}=2 \boldsymbol{y}^{T}(\mathbf{D}-\mathbf{W}) \boldsymbol{y}=2 \boldsymbol{y}^{T} \mathbf{L} \boldsymbol{y}
$$

where $\mathbf{D}$ is a diagonal matrix whose entries are $D_{i i}=\sum_{j} W_{i j}$, and $\mathbf{L}$ is the Laplacian Matrix [32]. Finally, the minimization problem of (1) equals to

$$
\min \boldsymbol{y}^{T} \mathbf{L} \boldsymbol{y} \quad \text { s.t. } \boldsymbol{y}^{T} \mathbf{D} \boldsymbol{y}=1 .
$$

The constraint $\boldsymbol{y}^{T} \mathbf{D} \mathbf{y}=1$ removes an arbitrary scaling factor in the embedding process. Note that $\mathbf{L}=\mathbf{D}-\mathbf{W}$, so that (3) equals to

$$
\max \boldsymbol{y}^{T} \mathbf{W} \boldsymbol{y} \quad \text { s.t. } \boldsymbol{y}^{T} \mathbf{D} \boldsymbol{y}=1 .
$$

The optimal $\boldsymbol{y}$ can be obtained by solving the following maximum eigenproblem:

$$
\mathrm{W} y=\lambda \mathrm{D} y
$$

For classification purposes, a mapping for all samples, including new testing samples, is required. Suppose that $\boldsymbol{\alpha}$ is a 


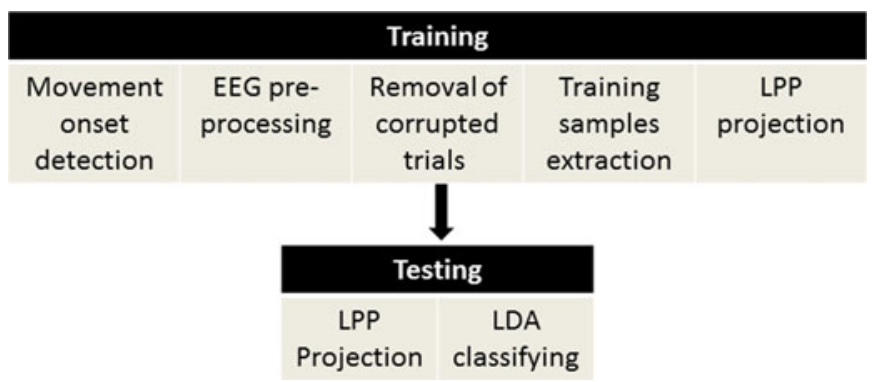

Fig. 2. Algorithm Flow. The LPP-LDA classifier was trained offline using the data obtained from the training session, and then applied in the online testing session.

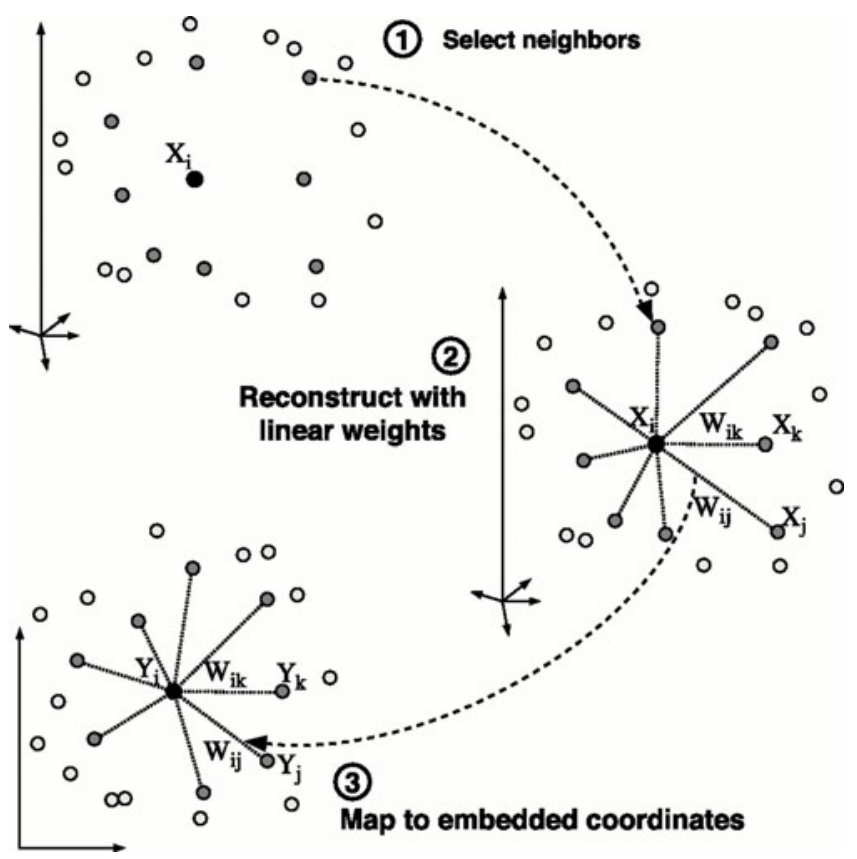

Fig. 3. Three steps for LPP (adapted from [33], with permission to reproduce). Step 1: select neighbors for each sample, i.e. $\boldsymbol{X}_{i}$ in original high-dimensional space; step 2: reconstruct $\boldsymbol{X}_{i}$ with linear weights $\boldsymbol{W}_{i j}$, which represent the distance between $\boldsymbol{X}_{i}$ and its neighbors, such as $\boldsymbol{X}_{j}$; step 3: project $\boldsymbol{X}_{i}$ to the embedded coordinates, i.e. $\boldsymbol{Y}_{i}$ in the reduced-dimensional space.

transformation vector, that is $\boldsymbol{y}^{T}=\boldsymbol{\alpha}^{T} \boldsymbol{X}$, then the above eigenproblem can be transformed into

$$
\mathrm{XWX}^{T} \boldsymbol{\alpha}=\lambda \mathrm{XDX}^{T} \boldsymbol{\alpha} .
$$

As illustrated in Fig. 3, there are three steps for LPP [33], [34]: first, the neighbors are selected to each sample of $X$ in the original high-dimensional space, after which the linear weights matrix $\mathbf{W}$, i.e., the distance between each sample of $\boldsymbol{X}$ and its neighbors, is calculated. Finally $\boldsymbol{X}$ is projected to $\boldsymbol{y}$, which is in the reduced-dimensional space.

\section{LPP-LDA Processing Steps}

There were five processing steps in the training stage of the signal processing (see Fig. 2):

1) Movement Onset Detection: The Teager-Kaiser energy operator was used here to detect movement (self-paced dorsi-

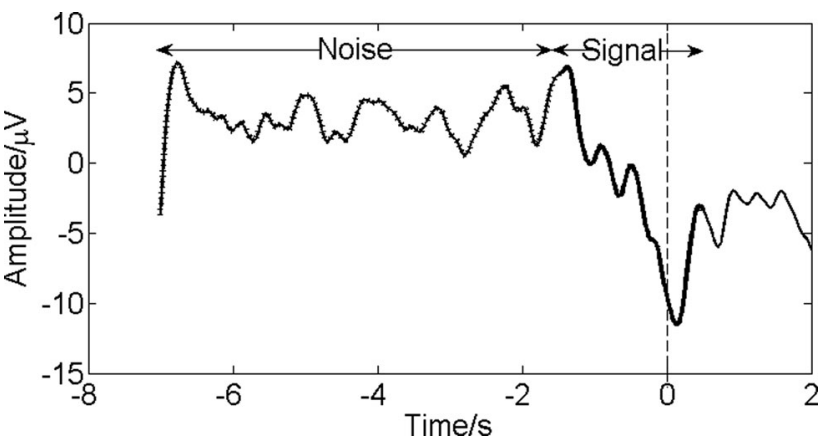

Fig. 4. Single trial MRCP of self-paced dorsiflexion in training run $(n=1)$ Time zero is when the dorsiflexion was executed, as indicated by the dashed vertical line. For LPP training set, the signal from -1.5 to $0.5 \mathrm{~s}$ was taken as "signal portion," while others was taken as "noise portion."

flexion) onsets from EMG online, which was shown to be more accurate than using the amplitude of the surface EMG [35].

2) EEG Preprocessing: A band-pass filter from 0.05 to $3 \mathrm{~Hz}$ and the large Laplacian spatial filter centered at $\mathrm{Cz}$ were used to enhance the signal-noise ratio of $\mathrm{Cz}$. This virtual $\mathrm{Cz}$ was used in subsequent processing steps.

3) Removal of Corrupted Trials: After the first two steps, $2.5 \mathrm{~s}$ before and $1.5 \mathrm{~s}$ after each movement onset of the preprocessed EEG, i.e., the virtual channel at $\mathrm{Cz}$, were presented to the experimenter immediately after each movement execution. Due to the subject's involuntary movement, such as eye blinking or jaw clutching during the training procedure, some trials were clearly corrupted, e.g., inconsistent morphology, or abnormal peak-to-peak amplitude $(<5 \mathrm{uV}$ or $>30 \mathrm{uV})$. These trials were flagged by the experimenter, so that they could be excluded from subsequent processing.

4) Training Samples Extraction: The data between $1.5 \mathrm{~s}$ before and $0.5 \mathrm{~s}$ after the movement onset of virtual $\mathrm{Cz}$ were extracted as the "signal portion," while EEG data between $1.5 \mathrm{~s}$ before this onset and $5 \mathrm{~s}$ after the previous onset were extracted as the "noise portion" (see Fig. 4). Thus, the two-class training set $\boldsymbol{X}_{M \times N}$ was compiled for the LPP-LDA classifier. $\boldsymbol{N}$ was the number of training samples, while $M$ was the length of them.

5) LPP-LDA Classification: The LPP-LDA classifier was trained offline using the training set $\boldsymbol{X}_{M \times N}$. The training set $\boldsymbol{X}_{M \times N}$ was projected to a lower dimensional feature space $\boldsymbol{y}_{K \times N}^{T}$ (LPP space) using LPP transform vector $\boldsymbol{\alpha}_{K \times M}^{T} \cdot \boldsymbol{K}$ was the reduced dimension by LPP. And LDA was used to classify the two classes in the LPP space $\boldsymbol{y}_{K \times N}^{T}$. As for any dimension reduction algorithms, the optimal dimension for the LPP space $\boldsymbol{y}_{K \times N}^{T}$ needs to be determined to achieve optimal performance. In the current study, the optimal dimension for LPP was determined through a pilot investigation, in which four healthy subjects (age: $30.5 \pm 5.1 \mathrm{yrs}$ ) participated only in one session identical to the training session of the main protocol. The threefold cross validation was applied on training data offline, with the dimension of the LPP varying from 1 to the dimension of the data. The optimal dimension was chosen based on the results of the pilot investigation (see Section III-A), and applied to the online experiment. Alternatively, it would have been also possible to obtain a subject-specific optimal dimension based on 
the individual training dataset. We preferred to find a common value for all subjects to reduce the number of parameters, thus making the algorithm more general across subjects.

The trained classifier was then applied online in the testing session. The incoming data $x_{M \times 9}^{i}$ (2-s windows with $0.1 \mathrm{~s}$ increments) was preprocessed in the same way as the training data [see Section II-D.2)]. Then, each preprocessed data segment $x_{M \times 1}^{i^{\prime}}$ was projected to the LPP space in real time, and the trained LDA classified each segment into one of the two classes. When two consecutive decisions were classified as "signal," an MRCP detection would be generated and a red bar in the subjectview GUI would be presented immediately.

\section{E. MF Processing}

The details of the MF approach were presented in [13]. A template of MRCP, namely $h$, is first obtained from the training data as the epoch average of all training trials. In the testing session, denoting the incoming sequence as $x$, the output of MF is defined as

$$
f[n]=(h * x)[n]=\sum_{-\infty}^{\infty} h[n-k] x[k]
$$

where $f$ is the likelihood ratio between $h$ and $x$. The incoming sequence would be detected as "signal" when $f$ is above the preset threshold, and otherwise as "noise."

The MF training procedure was identical to LPP-LDA training from step 1 to step 4 (see Section II-D). The MF only utilized the signal portion, from which an MRCP template $h_{M \times 1}$ was extracted. A threefold cross validation was applied to the training data, and the threshold of MF was chosen at the midpoint of the turning phase of the receiver operating characteristic (ROC) curve [13].

In the online testing session of MF, the incoming data $x_{M \times 9}^{i}$ was preprocessed, as done for the LPP-LDA. Then, the likelihood ratio between the preprocessed incoming data $x_{M \times 1}^{i^{\prime}}$ and the template $h_{M \times 1}$ was computed [13]. When two consecutive windows were above the MF threshold, a red bar in the subjectview GUI would be presented immediately, indicating an MRCP detection.

\section{F. Statistical Analysis}

Due to the limited number of subjects, a nonparametric test (Mann-Whiteney U Test) was used to analyze significant differences among the performance of the BCI when varying the method for detection and the type of task. The response variable of the Mann-Whiteney U Test included TPR, aFP, pFP, and DL. The two factors of the test were: tasks (two levels: execution and imagery) and algorithms (two levels: LPP-LDA and MF). For all tests, the significance level was set to 0.05 .

\section{RESULTS}

\section{A. Offline Analysis}

Offline analysis was performed on the data of the pilot investigation on four subjects, which were used to determine the optimal dimension for the LPP space.

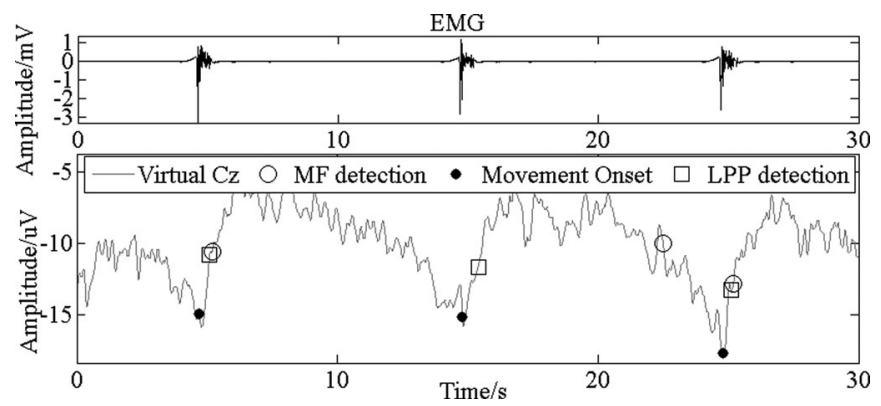

Fig. 5. Representative detection output by MF and LPP-LDA on the same training data in three-fold cross validation. Filled cycles stand for the movement onsets detected from EMG; empty cycles are the MF detections, while squares are detections by LPP-LDA.

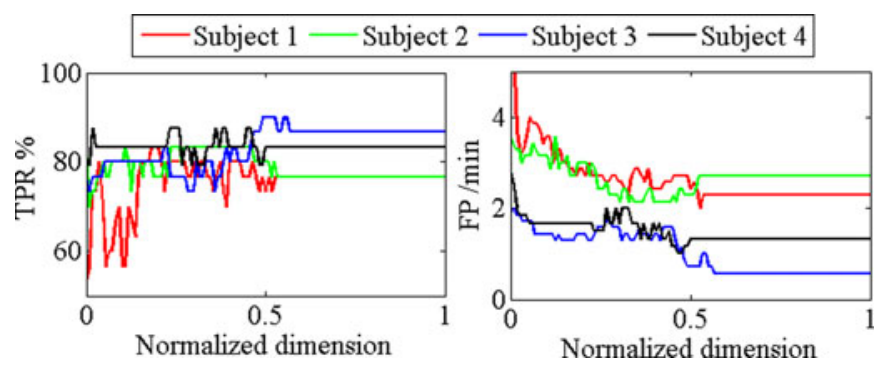

Fig. 6. Relationship between the LPP dimension and detection accuracy. Lines with different colors represent results from four subjects. Solid lines stand for TPR, while dash lines are TP. The LPP dimension was normalized to 0-1 with respect to the total dimension of the respective training data.

1) Optimal Dimension of the LPP Space: The optimal LPP dimension was chosen on the basis of the analysis of the data from the four subjects participating in the pilot experiment. As shown in Fig. 6, there were typical conditions in which the detection accuracy (i.e., TPR and FP) changed with respect to the dimension of the LPP space. For the four subjects of the pilot study, the TPR increased gradually while FP decreased rapidly when the dimension increased from 0 to approximately $60 \%$ of the original dimension, whereas these two indexes were approximately constant for greater dimensions. Based on these observations in the pilot study, the dimension of the LPP space in all subsequently analysis was set to $60 \%$ of the original dimension.

2) Representative Detection Output on the Training Data: Fig. 5 illustrates a representative example of the output of the two algorithms on the same training data in threefold crossvalidation. LPP-LDA was capable of identifying true MRCPs yet rejecting non-MRCP signal segments with very similar morphology. In contrast, the MF was shown to be easily influenced by small fluctuations, and missed true signals when the morphology changed.

\section{B. Online Performance}

The results in this section, as shown in Tables I and II, are based on the online testing session.

The Mann-Whiteney U Test showed that there was no statistical significance between execution and imagery with MF for TPR, aFP, pFP and DL ( $p=0.18,0.59,0.75$, and 0.064, respectively). Same results were obtained with LPP-LDA ( $p=0.15$, 
TABLE I

DETECTION ACCURACY WITH LPP-LDA AND MF

\begin{tabular}{|c|c|c|c|c|c|c|c|c|c|c|c|c|}
\hline \multirow{3}{*}{ Subject } & \multicolumn{6}{|c|}{ LPP-LDA } & \multicolumn{6}{|c|}{ MF } \\
\hline & \multicolumn{2}{|c|}{ TPR\% } & \multicolumn{2}{|c|}{ aFP/min } & \multicolumn{2}{|c|}{ pFP } & \multicolumn{2}{|c|}{ TPR\% } & \multicolumn{2}{|c|}{ aFP/min } & \multicolumn{2}{|c|}{ pFP } \\
\hline & Exec & Imag & Exec & Imag & Exec & Imag & Exec & Imag & Exec & Imag & Exec & Imag \\
\hline 1 & 90 & 93 & 1.9 & 0.0 & 1 & 2 & 60 & 67 & 2.2 & 2.9 & 1 & 1 \\
\hline 2 & 95 & 90 & 0.7 & 0.6 & 1 & 0 & 90 & 55 & 0.7 & 1.2 & 1 & 4 \\
\hline 3 & 87 & 60 & 1.6 & 1.8 & 1 & 4 & 73 & 60 & 0.9 & 3.0 & 1 & 2 \\
\hline 4 & 80 & 73 & 2.6 & 2.1 & 6 & 3 & 75 & 67 & 3.5 & 3.8 & 5 & 7 \\
\hline 5 & 85 & 87 & 0.4 & 0.8 & 2 & 4 & 85 & 47 & 3.8 & 4.4 & 6 & 8 \\
\hline 6 & 85 & 55 & 1.5 & 1.4 & 0 & 2 & 65 & 60 & 0.6 & 1.2 & 3 & 2 \\
\hline 7 & 60 & 67 & 2.8 & 2.3 & 5 & 0 & 65 & 67 & 2.3 & 1.9 & 0 & 5 \\
\hline 8 & 90 & 73 & 1.5 & 2.0 & 1 & 5 & 70 & 80 & 2.0 & 3.5 & 3 & 7 \\
\hline 9 & 85 & 75 & 0.7 & 0.0 & 0 & 0 & 65 & 67 & 1.7 & 2.3 & 4 & 3 \\
\hline Mean \pm SD & \multicolumn{2}{|c|}{$79 \pm 12$} & \multicolumn{2}{|c|}{$1.4 \pm 0.8$} & \multicolumn{2}{|c|}{$2.1 \pm 1.9$} & \multicolumn{2}{|c|}{$68 \pm 10$} & \multicolumn{2}{|c|}{$2.3 \pm 1.1$} & \multicolumn{2}{|c|}{$3.5 \pm 2.3$} \\
\hline
\end{tabular}

TPR is the ratio of the number of true detections and total attempts; while aFP and pFP are the number of false detections in active phase and passive phase, respectively. Exec and Imag represent execution phase and imagery phase, respectively.

TABLE II

DETECTION LATENCY (ms) WITH LPP-LDA AND MF

\begin{tabular}{c|cc|cc}
\hline \hline \multirow{2}{*}{ Subjects } & \multicolumn{2}{|c|}{ LPP-LDA } & \multicolumn{2}{c}{ MF } \\
\cline { 2 - 5 } & Exec & Imag & Exec & Imag \\
1 & 574 & 466 & 560 & 478 \\
2 & 140 & 220 & 571 & 521 \\
3 & 241 & 506 & 480 & 605 \\
4 & 223 & 277 & 453 & 374 \\
5 & 70 & 212 & 92 & 356 \\
6 & 289 & 544 & 571 & 514 \\
7 & 63 & 600 & 560 & 288 \\
8 & 143 & 331 & 352 & 492 \\
9 & 368 & 405 & 517 & 492 \\
\hline Mean \pm SD & \multicolumn{3}{|c|}{$\mathbf{1 6 5}$} & $\mathbf{4 6 0} \pm \mathbf{1 2 3}$ \\
\hline \hline
\end{tabular}

$0.20,0.16$, and 0.59 , respectively). Following these results, the data from the two tasks were pooled together.

Subsequently, the Mann-Whiteney U Test was applied on the pooled data to analyze the significance between two algorithms. TPR with LPP-LDA was found to be significantly $(p=0.007)$ higher than that with MF $(79 \pm 12 \%$ versus $68 \pm 10 \%)$. LPP-LDA was found to produce significantly $(p=0.016)$ lower aFP than those with MF ( $1.4 \pm 0.8 / \mathrm{min}$ versus $2.3 \pm 1.1 / \mathrm{min})$. LPP-LDA also produced significantly shorter DL $(p=0.013)$ than MF ( $315 \pm 165 \mathrm{~ms}$ versus $460 \pm 123 \mathrm{~ms})$. However, there was no significance ( $p=0.064$ ) for $\mathrm{pFP}$ between the two algorithms, although it trended to be less with LPP-LDA than MF $(2.1 \pm 1.9$ versus $3.5 \pm 2.3)$.

\section{DisCUSSION}

In this study, a manifold based nonlinear dimension reduction method (LPP) was applied, along with LDA, to perform online detection of MRCPs from EEG signals in execution and imagery of dorsiflexions. Across all subjects, the proposed algorithm had similar FP for execution and imagination. The respective TPR for execution and imagery was $>80 \%$ and $>70 \%$.. These results were significantly better than those with the MF approach pre- viously reported in the literature [13], [14], [23]. Furthermore, the DL ( $315 \pm 165 \mathrm{~ms})$ was also significantly shorter than that with MF.

\section{A. Motor Intention Detection and Closed-Loop BCI Control}

The robust detection of motor intention is an essential and critical issue for the development of self-paced close-loop BCI control systems. For BCI control applications, the acceptable delay in control has not been investigated in detail but, in other fields, e.g., multifunction prostheses control by myoelectric signals, a 200-ms delay is considered as acceptable [36]-[38]. For the applications of BCI-based neurorehabilitation to induce plasticity, it was demonstrated that the necessary delay was in the same range as for control, i.e., in the order of a few hundred millisecond [6]. Therefore, a reliable detection with high accuracy and minimal latency would play an important role in an effective BCI rehabilitation tool. In the current study, the latency was in $200-400 \mathrm{~ms}$, making it ideal for a close-loop BCI control system.

In the past decades, SMR has been used to detect motor intention in studies, in which BCIs were used to control visual feedback [12] or trigger external devices [39]. However, it is difficult for naïve subjects to use an SMR-based BCI system, since a rather long (in the order of weeks) training session is necessary before a reasonably detection accuracy can be attained [40]. In addition, the latency was not investigated in these studies. This may explain why weeks of intervention were required for inducing plasticity [11], as the association of the Hebbian rule was not established when the afferents arrived at the cortex with too large delay with respect to the movement intentions or attempts [41].

In recent years, slow cortical potentials attracted the attention in the rehabilitation field. Garipelli et al. investigated the effect of different band-pass filter in the detection accuracy of CNV, yielding $0.88 \pm 0.05$ of area under curve in ROC with the optimal filter in an offline study [19]. Also in an offline study, Ahmadian et al. used constrained blind source extraction and showed that there was a tradeoff between TPR and FP [42]. These studies investigated upper limb movements, which 
arguably have more distinctive spatial pattern than dorsiflexion. As such, it is difficult to compare the results of these two recent offline studies with the results from the current online study with lower limb movement. More importantly, the latency was not investigated in these previous studies, although it is a crucial determinant of the efficacy of a rehabilitation training based on this type of BCI switches [6].

In a series of studies by our group, we demonstrated that motor intention could be detected from MRCP using the MF, with satisfactory accuracy and small latency [13], [14], [23]. When such a detection was used to trigger electrical stimulation, plasticity was induced with a short intervention ( $\sim 30 \mathrm{~min})$, and outlasted the intervention [14]. In the current study, LPP-LDA showed higher accuracy and shorter latency than MF, and thus would play an important role in further enhancing the efficacy of this BCI-driven rehabilitation approach that is uniquely designed to induce plasticity specific to the target muscle. Preliminary verification of this hypothesis has been recently reported in abstract form in a study in which we used the LPP-LDA detection approach proposed in this study to trigger an ankle foot orthosis [43]. In that study, plasticity was induced with an even shorter intervention time and more effectively with the algorithm proposed in this study than in previous investigations with MF detector.

\section{B. Advantages of LPP}

1) Robust Performance: The LPP-LDA yielded a significantly higher accuracy, compared with MF (see Table I). In addition, passive FP was tested in this study, and LPP-LDA also showed its superior ability in reducing FP when the subjects were in idle condition.

More importantly, for LPP-LDA, there was no significant difference between the FP (both aFP and pFP) in both tasks. This insensitivity to task types demonstrated the robustness of LPP-LDA.

There are two reasons why LPP-LDA outperformed MF. One lies in the data organization. For MF, the MRCP template was extracted from the epoch average of all trials of "signal portion" in the training session. This process practically dropped the specific characters of MRCP in different trials, and also discarded the direct information from "noise portion," even though it was implicitly used during the manual adjustment of the threshold. On the contrary, both signal portion (MRCP) and noise portion were used to train the LPP-LDA classifier, which thus made full use of the available information. Another reason is the powerful ability of LPP in preserving the intrinsic structure of the data in its original high dimensional space. That is why it could detect MRCPs when its morphology was changed, and reject false detections induced by signal fluctuation, while MF cannot (see Fig. 5).

Moreover, LPP-LDA showed shorter latency than MF. The DL with LPP-LDA was $\sim 300 \mathrm{~ms}$, which was significantly shorter than that with $\mathrm{MF}$ ( $\sim 500 \mathrm{~ms})$.

It should be noted that we used the signal of $0.5 \mathrm{~s}$ after the movement onset as part of the templates for both detectors, because the rebound phase (movement monitoring potential) is highly distinctive in many subjects. The DL could be further de- creased by choosing a shorter portion of the MRCP as template, at the expense of detection accuracy.

2) Subject Independent Parameters: The threshold of MF output needs to be optimized. When the threshold is too low, a small variation would result in an MRCP detection, leading to high FP. On the contrary, the TPR would be low if the threshold was set too high. That is why there is a tradeoff between the TPR and FP. The optimization step is usually realized by a cross validation of the training data on individual basis [13]. However, two issues should be treated carefully during this process. One is how to define the optimal parameters. There is a balance between the TPR and FP, but it is still not clear which one should have priority. Another issue is that the optimal parameter could be changed as the morphology of MRCP changes during different types of task (i.e., execution or imagery), and likely need to be calibrated from session to session. In the current study, even though the optimal parameter was chosen for the execution tasks, it may not be optimal for imagination tasks.

For LPP-LDA, the parameter that needs to be optimized is the dimension of the LPP space. As we demonstrated in Fig. 6, when the dimension was reduced to close to $60 \%$ of the total dimension of the training data, the performance of LPP-LDA kept consistent across subjects.

It is also possible for BCI applications to set subject specific and session specific parameters. However, the calibration process for optimizing the parameters implies a longer intervention protocol, which could be problematic for users, such as stroke patients. Therefore, the subject-independent property of LPP-LDA is especially desirable for BCI system tailored to neurorehabilitation applications.

\section{Potential Applications}

As demonstrated in this study, LPP led to very good performance for a brain switch with high TPR, low FP, short DL, good robustness with respect to the MRCP morphology, and subjectindependent parameters. It could be a promising tool in BCI applications. One of these foreseeable applications lies in the BCI driven external device for neurorehabilitation [14], [15]. It can play an important role in the development of an effective and versatile BCI system in inducing plasticity.

\section{Limitations}

In the current study, we collected EEG data of 30 trials of real movement of dorsiflexion as the training data for both the MF and LPP-LDA, and it showed desirable performance. LPP's performance in general increases with the size of the training data, as repeatedly demonstrated in the literature on image processing [44], [45]. Our preliminary analyses showed that the classifier would not work when the training trials were less than 15. However, the influence of the number of trials on performance for MRCP detection is not systematically investigated. Moreover, for patients who lost complete motor function of the target limb and thus cannot produce detectable EMG, as is many cases in neurorehabilitation applications, it would not be possible to use real movements for training. In this case, cue-based (synchronous) imagery training would be necessary and this would increase the number of training trials since MRCPs from 
motor imagery have lower signal to noise ratio than those from real motor tasks [25].

Currently, the corrupted trials were identified and manually removed from the training session. However, this step should be executed automatically in the future.

In addition, the target users of the envisioned closed-loop BCI rehabilitation system, i.e., patients with motor disorders, such as stroke, were not involved in the current study. Previous studies using MF reported similar results in both healthy subjects and patients with MF [13], [27], indicating the potential of using MRCP in the patient population. However, the exact performance of the proposed LPP-LDA in patient population needs further investigation.

\section{CONCLUSION}

We proposed an LPP-LDA classifier for detecting MRCPs in real time, and compared its performance with the standard MF in an online experiment. We demonstrated that LPP-LDA performed significantly better than MF, with higher accuracy and lower latency. In addition, it showed the desirable property of subject-independent parameters. Based on these results, the proposed brain switch is an extremely promising tool for the development of user-intention-driven closed-loop BCI neurorehabilitation systems.

\section{REFERENCES}

[1] J. J. Shih, D. J. Krusienski, and J. R. Wolpaw, "Brain-computer interfaces in medicine," Mayo Clin. Proc., vol. 87, pp. 268-279, 2012.

[2] J. J. Daly and J. R. Wolpaw, "Brain-computer interfaces in neurological rehabilitation," Lancet Neurol., vol. 7, pp. 1032-1043, 2008.

[3] F. Galán, M. Nuttin, E. Lew, P. W. Ferrez, G. Vanacker, J. Philips, and J. D. R. Millán, "A brain-actuated wheelchair: Asynchronous and noninvasive brain-computer interfaces for continuous control of robots," Clin. Neurophysiol., vol. 119, pp. 2159-2169, 2008.

[4] N. Mrachacz-Kersting, I. K. Niazi, N. Jiang, A. M. Pavlovic, S. Radovanovic, V. Kostic, D. Popovic, K. Dremstrup, and D. Farina, "A novel brain-computer interface for chronic stroke patients," in Converging Clinical and Engineering Research and Neurorehabilitation, Eds. J. Pose, D. Torricelli and M. Pajaro, pp. 837-841, Springer Berlin Heidelberg, 2013.

[5] N. Jiang, N. Mrachacz-Kersting, R. Xu, K. Dremstrup, and D. Farina, "An accurate, versatile, and robust brain switch for neurorehabilitation," in The Annu. BCI Res. Award, 2013.

[6] N. Mrachacz-Kersting, "Precise temporal association between cortical potentials evoked by motor imagination and afference induces cortical plasticity," J. Physiol., vol. 590, pp. 1669-1682, Apr. 2012.

[7] G. Pfurtscheller and F. H. Lopes Da Silva, "Event-related EEG/MEG synchronization and desynchronization: Basic principles," Clin. Neurophysiol., vol. 110, pp. 1842-1857, 1999.

[8] N. Birbaumer, "Slow cortical potentials: Their origin, meaning, and clinical use," Brain Behav. Past, Present Future, pp. 25-39, 1997.

[9] G. Pfurtscheller and T. Solis-Escalante, "Could the beta rebound in the EEG be suitable to realize a 'brain switch'?" Clin. Neurophysiol., vol. 120, no. 1, pp. 24-29, Jan. 2009.

[10] G. Müller-Putz and V. Kaiser, "Fast set-up asynchronous brain-switch based on detection of foot motor imagery in 1-channel EEG," Med. Biol. Eng. Comput., vol. 48, no. 3, pp. 229-233, Mar. 2010.

[11] F. Pichiorri, F. De Vico Fallani, F. Cincotti, F. Babiloni, M. Molinari, S. C. Kleih, C. Neuper, A. Kübler, and D. Mattia, "Sensorimotor rhythmbased brain-computer interface training: The impact on motor cortical responsiveness," J. Neural Eng., vol. 8, p. 025020, 2011.

[12] A. Ramos-Murguialday, D. Broetz, M. Rea, L. Läer, O. Yilmaz, F. L. Brasil, G. Liberati, M. R. Curado, E. Garcia-Cossio, A. Vyziotis, W. Cho, M. Agostini, E. Soares, S. Soekadar, A. Caria, L. G. Cohen, and N. Birbaumer, "Brain-machine interface in chronic stroke rehabilitation: A controlled study," Ann. Neurol., vol. 74, pp. 100-108, 2013.
[13] I. K. Niazi, N. Jiang, O. Tiberghien, J. F. Nielsen, K. Dremstrup, and D. Farina, "Detection of movement intention from single-trial movementrelated cortical potentials," J. Neural Eng., vol. 8, p. 066009, 2011.

[14] I. K. Niazi, N. Mrachacz-Kersting, N. Jiang, K. Dremstrup, and D. Farina, "Peripheral electrical stimulation triggered by self-pace detection of motor intention enhances motor evoked potentials," IEEE Trans. Neural Rehabil. Syst. Eng., vol. 20, no. 4, pp. 595-604, Jul. 2012.

[15] N. Mrachacz-Kersting, I. K. Niazi, N. Jiang, A. M. Pavlovic, Radovanović, V. S., Kostic, and D. Farina, "A novel brain-computer interface for chronic stroke patients," in Converging Clinical and Engineering Research on Neurorehabilitation. Berlin, Germany: Springer, 2013, pp. 837-841.

[16] J. Tecce, "Contingent negative variation (CNV) and psychological processes in man," Psychol. Bull., vol. 77, pp. 73-108, 1972.

[17] H. Kornhuber and L. Deecke, "Changes in the brain potential in voluntary movements and passive movements in man: Readiness potential and reafferent potential," Pflugers Arch. Gesamte Physiol. Menschen Tiere, vol. 10, no. 284, pp. 1-17, 1965.

[18] G. Gangadhar, R. Chavarriaga, and J. Millan, "Fast recognition of anticipation-related potentials," IEEE Trans. Biomed. Eng., vol. 56, no. 4, pp. 1257-1260, Apr. 2009.

[19] G. Garipelli, R. Chavarriaga, and J. Millan, "Single trial analysis of slow cortical potentials: A study on anticipation related potentials," J. Neural Eng., vol. 10, p. 036014, 2013.

[20] O. Bai, P. Lin, S. Vorbach, and J. Li, "Exploration of computational methods for classification of movement intention during human voluntary movement from single trial EEG," Clin. Neurophysiol., vol. 118, pp. 2637$2655,2007$.

[21] T. Bradberry, "Reconstructing three-dimensional hand movements from noninvasive electroencephalographic signals," J. Neurosci., vol. 30, pp. 3432-3437, 2010.

[22] E. Lew, R. Chavarriaga, S. Silvoni, and J. Millan, "Detection of self-paced reaching movement intention from EEG signals," Front. Neuroeng., vol. 5, p. 13, 2012.

[23] I. K. Niazi, N. Jiang, M. Jochumsen, J. F. Nielsen, K. Dremstrup, and D. Farina, "Detection of movement-related cortical potentials based on subject-independent training," Med. Biol. Eng. Comput., vol. 51, pp. 507$512,2013$.

[24] X. He and P. Niyogi, "Locality preserving projections," in Proc. Neural Inform. Process. Syst., 2003, pp. 153-160.

[25] O. F. do Nascimento, K. D. Nielsen, and M. Voigt, "Movement-related parameters modulate cortical activity during imaginary isometric plantarflexions," Exp. Brain Res., vol. 171, pp. 70-90, 2006.

[26] X. Zhang and P. Zhou, "Sample entropy analysis of surface EMG for improved muscle activity onset detection against spurious background spikes," J. Electromyogr. Kinesiol., vol. 22, pp. 901-907, 2012.

[27] N. Mrachacz-Kersting, N. Jiang, K. Dremstrup, and D. Farina, "Coupling of motor imagination and nervous system stimulation to induce cortical plasticity," in Proc. 5th Int. Conf. Non-invasive Brain Stimulation, 2013.

[28] M. Johanshahi and M. Hallettm, Eds., The Bereitschaftspotential: Movement-Related Cortical Potentials. Berlin, Germany: Springer, 2003.

[29] D. J. McFarland, C. W. Anderson, K.-R. Müller, A. Schlögl, and D. J. Krusienski, "BCI meeting 2005-workshop on BCI signal processing: Feature extraction and translation," IEEE Trans. Neural Syst. Rehabil. Eng., vol. 14, no. 2, pp. 135-138, Jun. 2006.

[30] N. Ince, F. Goksu, A. Tewfik, and S. Arica, "Adapting subject specific motor imagery EEG patterns in space-time-frequency for a brain computer interface," Biomed. Signal Process. Control, vol. 4, no. 3, pp. 236-246, Jul. 2009.

[31] X. He, S. Yan, Y. Hu, P. Niyogi, and H.-J. Zhang, "Face recognition using laplacianfaces," IEEE Trans. Pattern Anal. Mach. Intell., vol. 27, no. 3, pp. 328-340, Mar. 2005.

[32] F. R. K. Chung, Spectral Graph Theory, in Cmbs Reg. Conf. Ser. Math (Book 92), American Mathematical Society, 1996.

[33] S. T. Roweis and L. K. Saul, "Nonlinear dimensionality reduction by locally linear embedding," Science, vol. 290, pp. 2323-2326, 2000.

[34] M. Belkin and P. Niyogi, "Laplacian eigenmaps and spectral techniques for embedding and clustering," in Proc. Neural Inform. Process. Syst., 2001, vol. 14, pp. 585-591.

[35] X. Li, P. Zhou, and A. S. Aruin, "Teager-kaiser energy operation of surface EMG improves muscle activity onset detection," Ann. Biomed. Eng., vol. 35, pp. 1584-1593, 2007.

[36] J. E. Paciga, P. D. Richard, and R. N. Scott, "Error rate in five-state myoelectric control systems," Med. Biol. Eng. Comput., vol. 18, pp. 287-290, 1980. 
[37] R. T. Lauer, P. H. Peckham, K. L. Kilgore, and W. J. Heetderks, "Applications of cortical signals to neuroprosthetic control: A critical review," IEEE Trans. Rehabil. Eng., vol. 8, no. 2, pp. 205-208, Jun. 2000.

[38] M. Velliste, S. Perel, M. C. Spalding, A. S. Whitford, and A. B. Schwartz, "Cortical control of a prosthetic arm for self-feeding," Nature, vol. 453, pp. 1098-1101, 2008.

[39] A. Biasiucci, R. Leeb, A. Al-Khodairy, V. Buhlmann, and J. Millan, "Motor recovery after stroke by means of BCI-guided functional electrical stimulation," in Proc. 5th Int. Brain-Comput. Interface Meeting, 2013.

[40] E. Buch, C. Weber, L. G. Cohen, C. Braun, M. A. Dimyan, T. Ard, J. Mellinger, A. Caria, S. R. Soekadar, A. Fourkas, and N. Birbaumer, "Think to move: A neuromagnetic brain-computer interface (BCI) system for chronic stroke," Stroke, vol. 39, pp. 910-917, 2008.

[41] D. O. Hebb, "The organization of behavior: A neuropsychological theory," vol. 44, p. 335, 1949.

[42] P. Ahmadian, S. Sanei, and L. Ascari, "Constrained blind source extraction of readiness potentials from EEG," IEEE Trans. Neural Syst. Rehabil. Eng., vol. 21, no. 4, pp. 567-575, Jul. 2013.

[43] R. Xu, N. Jiang, G. Asín, J. C. Moreno, J. L. Pons, N. Mrachacz-Kersting, and D. Farina, "An ambulatory BCI-driven orthosis for stroke rehabilitation," in Proc. 5th Int. Brain-Comput. Interface Meeting, 2013.

[44] Q. You, N. Zheng, S. Du, and Y. Wu, "Neighborhood discriminant projection for face recognition," Pattern Recog. Lett., vol. 28, no. 10, pp. 11561163, 2007.

[45] P. Y. Han, A. T. B. Jin, and F. S. Abas, "Neighbourhood preserving discriminant embedding in face recognition," J. Vis. Commun. Image Represent., vol. 20, no. 8, pp. 532-542, 2009.

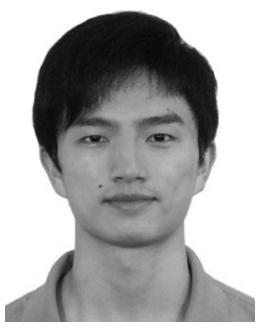

Ren Xu (S'12) received the B.Sc. degree in electronic information science and technology from the University of Science and Technology of China, Hefei, China, and the M.Sc. degree in signal and information processing from Chinese Academy of Sciences, Beijing, China, in 2009 and 2012, respectively. He is currently working toward the Ph.D. degree in applied computer science at University Medical Center, Georg-August University, Göttingen, Germany.

His research interests include biomedical signal processing and brain-computer interface for

rehabilitation.

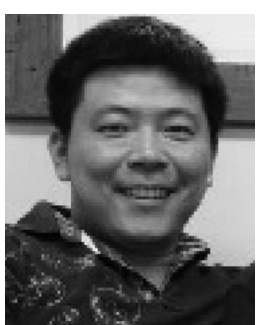

Ning Jiang (S'02-M'09) received the B.S. degree in electrical engineering from Xi'an Jiaotong University, Xi' an, China, in 1998, and the M.Sc. and Ph.D. degrees in engineering from the University of New Brunswick, Fredericton, NB, Canada, in 2004 and 2009 , respectively.

He is currently a Research Scientist with the Department of Neurorehabilitation Engineering, University Medical Center Göttingen, Georg-August University, Göttingen, Germany. His research interests include signal processing of electromyography, advanced prosthetic control, neuromuscular modeling, and BCI for neurorehabilitation.

Dr. Jiang was a Marie Curie Fellow at the Strategic Technology Management, Otto Bock Healthcare GmbH, Germany from 2010 to 2012.

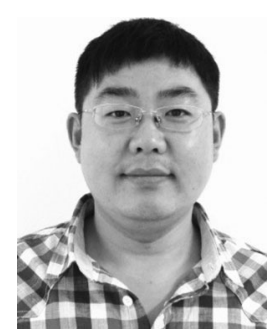

Chuang Lin received the M.Sc. and the Ph.D. degrees in signal processing from the Harbin Institute of Technology, Harbin, China, in 2004 and 2008, respectively.

$\mathrm{He}$ is an Assistant Professor in the Dalian University of Technology, Dalian, China. He is currently a Research Scientist of European Research Council Project DEMOVE in the Department of Neurorehabilitation Engineering, University Medical Center Göttingen, Georg-August University, Germany, within the Bernstein Center for Computational Neuroscience and the Bernstein Focus Neurotechnology Göttingen. His research interests include biomedical signal processing, stochastic signal processing, pattern recognition, and machine learning.

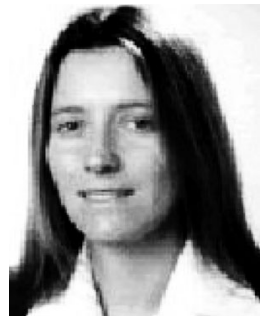

Natalie Mrachacz-Kersting received the M.Ed. degree in human movement science from the University of Western Australia, Perth, Australia, in 1997, and the $\mathrm{Ph} . \mathrm{D}$. degree in biomedical engineering from Aalborg University, Aalborg, Denmark, in 2005.

From 2005 to 2007, she has been a Lecturer at the Department of Sport and Exercise Science, the University of Auckland, Auckland, New Zealand and from 2007 to 2009, an Assistant Professor in Motor Control at Aalborg University, Aalborg, Denmark. Since 2009 she has been an Associate Professor at the Department of Health Science and Technology, Aalborg University, Aalborg, Denmark. There, she is the Head of two laboratories, the Motor Control Laboratory and the Neuroplasticity Laboratory. She acts as a Referee for numerous journals. Her main research interests include the role of feedback from muscle afferents in both motor control and neural plasticity.

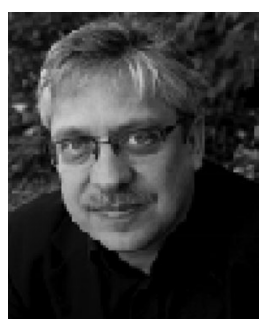

Kim Dremstrup (M'12) received the M.Sc., B.M.E, and $\mathrm{Ph} . \mathrm{D}$. degrees from Aalborg University, Aalborg, Denmark, in 1984 and 1993, respectively.

He is the Head of the Department of Health Science and Technology and a Professor within the Faculty of Medicine at Aalborg University, Aalborg, Denmark. He is affiliated to the Center for Sensory Motor Interaction where he is heading BCIlaboratory. His research interests include methods for retrieving and analysis of information from the brain. Especially methods for EEG-analysis, rehabilitation technology, brain-computer interfaces, and sleep research are points of foci. $\mathrm{He}$ was the inventor of the commercialized Nightingale Automatic Sleep Analyzer System and the coinventor of the widely used EDF bio-data format. $\mathrm{He}$ is the author and coauthor of more than 150 research publications including more than 50 peer reviewed journal papers.

Dr. Kim Dremstrup is the President for The Danish Society for Biomedical Engineering.

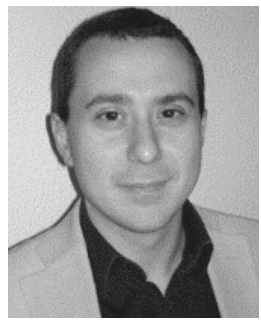

Dario Farina (M'01-SM'09) received the M.Sc. degree in electronics engineering from Politecnico di Torino, Torino, Italy, in 1998, and the Ph.D. degree in automatic control and computer science and in electronics and communications engineering from the Ecole Centrale de Nantes, Nantes, France, and Politecnico di Torino, respectively, in 2002

During 2002-2004, he was a Research Assistant Professor at Politecnico di Torino, and in 2004-2008 an Associate Professor in biomedical engineering at Aalborg University, Aalborg, Denmark. From 2008 to 2010, he was a Full Professor in motor control and biomedical signal processing, and the Head of the Research Group on neural engineering and neurophysiology of movement at Aalborg University. In 2010, he was appointed a Full Professor and the Founding Chair of the Department of Neurorehabilitation Engineering, University Medical Center Göttingen, Georg-August University, Germany, within the Bernstein Center for Computational Neuroscience. He is also the Chair for Neuroinformatics of the Bernstein Focus Neurotechnology Göttingen. His research interest include biomedical signal processing, specifically timefrequency analysis, pattern recognition, and blind source separation, modeling, neurorehabilitation technology, and neural control of movement. Within these areas, he has (co)-authored more than 250 papers in peer-reviewed journals and more than 300 among conference papers/abstracts, book chapters, and encyclopedia contributions. He is an Associate Editor of Medical and Biological Engineering and Computing and of the Journal of Electromyography and Kinesiology and member of the Editorial Board of the Journal of Neuroscience Methods.

Dr. Farina has been the Vice-President of the International Society of Electrophysiology and Kinesiology (ISEK) from 2010 to 2012 and is currently the President of ISEK. He received the 2010 IEEE Engineering in Medicine and Biology Society Early Career Achievement Award for his contributions to biomedical signal processing and to electrophysiology. He is an Associate Editor of IEEE TRANSACTIONS ON BIOMEDICAL ENGINEERING. 